Supporting information

\title{
Chiral 3D CdSe Nanotetrapods
}

\author{
Xiao Shao ${ }^{1,2, \dagger}$, Yue $W u^{1,2, \dagger}$, Shuang Jiang ${ }^{1 *}$, Bin Li $i^{1}$, Tianyong Zhang ${ }^{1^{*}}$ and Yong \\ $\operatorname{Yan}^{2,3 *}$
}

${ }^{1}$ Tianjin Key Laboratory of Applied Catalysis Science and Technology, School of Chemical Engineering and Technology, Tianjin University, Tianjin 300354, China ${ }^{2}$ CAS Key Laboratory of Nanosystem and Hierarchical Fabrication, CAS Center for Excellence in Nanoscience, National Center for Nanoscience and Technology, Beijing 100190, China

${ }^{3}$ University of Chinese Academy of Sciences, Beijing, 100049, China

$\dagger$ These authors contributed equally to this work.

*Corresponding authors' E-mail: shuangjiang@tju.edu.cn; tyzhang@,tju.edu.cn; yany@,nanoctr.cn 
Table S1 Summary of the size (length/width) and preparation condition of 3D CdSe nanotetrapods. Tp0 is the ZB CdSe seed.

\begin{tabular}{lllll}
\hline Sample & $\begin{array}{l}\text { Average arm length/width } \\
\text { of CdSe Tps (nm) }\end{array}$ & $\begin{array}{l}\mathbf{X}_{1}=\text { DDAB } \\
(\mathbf{m g})\end{array}$ & $\begin{array}{l}\mathbf{X}_{2}=\text { Injection } \\
\text { temperature }\left({ }^{\circ} \mathrm{C}\right)\end{array}$ & $\begin{array}{l}\mathbf{X}_{3}= \\
\text { Reaction } \\
\text { time }(\mathbf{m i n})\end{array}$ \\
\hline Tp0 & $5.62 / 5.62$ & 40 & 280 & 0.5 \\
\hline Tp1 & $15.70 / 5.68$ & 40 & 280 & 5 \\
Tp2 & $18.63 / 5.83$ & 70 & 300 & 5 \\
Tp3 & $21.99 / 6.03$ & 75 & 300 & 5 \\
Tp4 & $24.36 / 6.12$ & 85 & 300 & 10 \\
Tp5 & $28.03 / 6.34$ & 100 & 300 & 10 \\
\hline
\end{tabular}



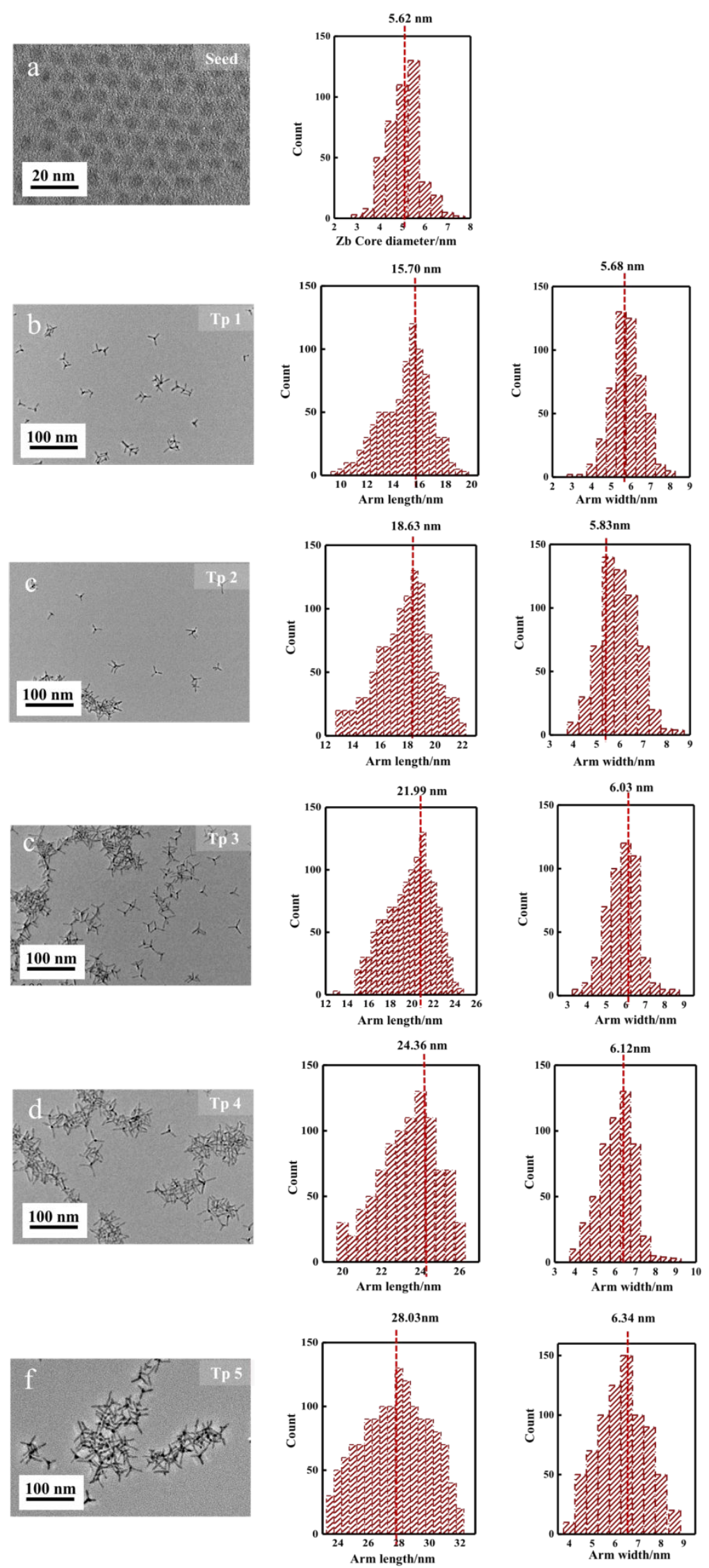

Fig. S1 TEM images (left) and histograms of arm width and length distributions (right) of CdSe core and Tps. 

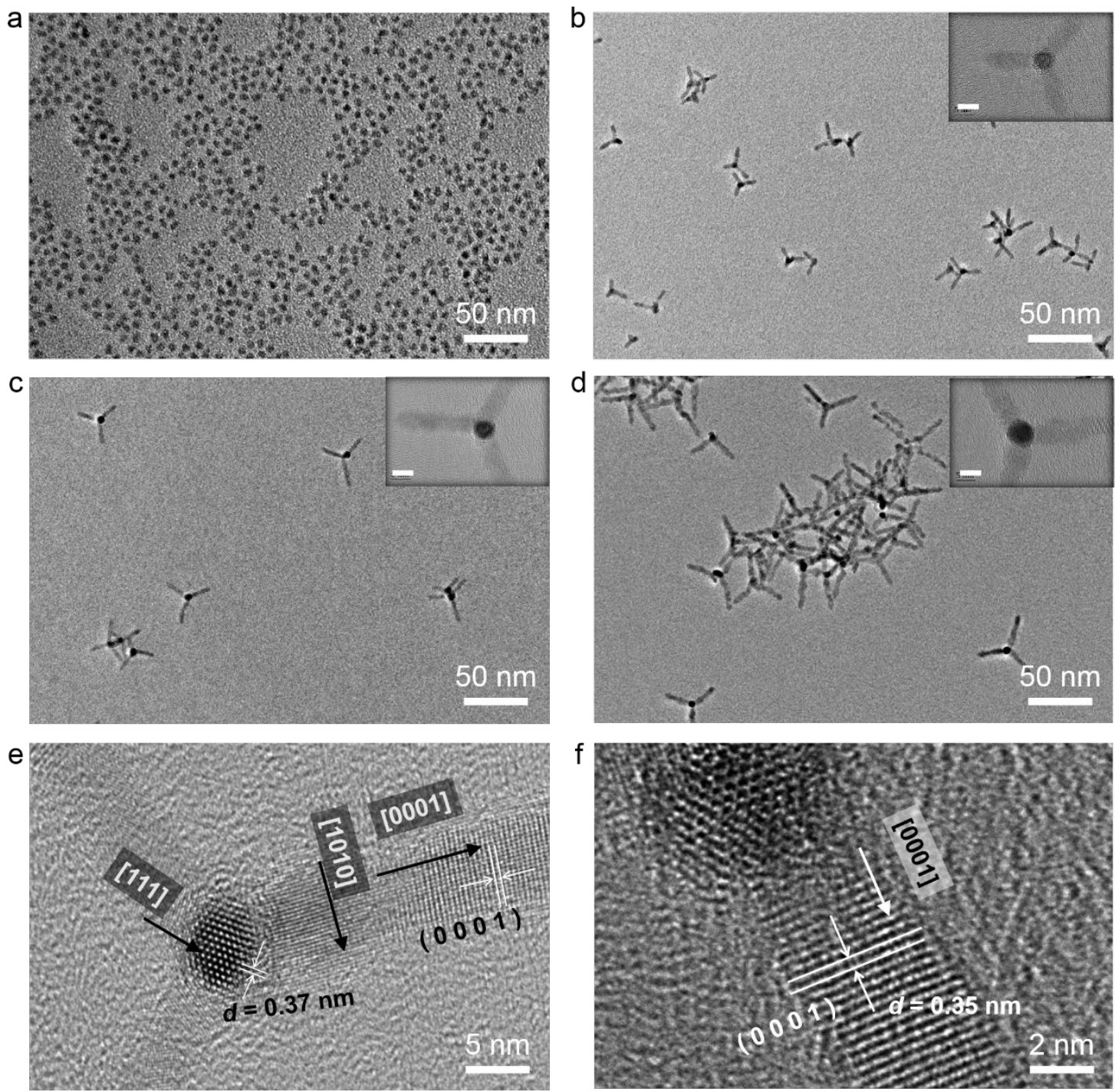

Fig. S2 TEM images of (a) ZB CdSe seed, Tps with different size (b) Tp1, (c) Tp3, and (d) Tp5. Insets: higher magnification of individual Tp. The scale bars were $5 \mathrm{~nm}$. (e) and (f) HRTEM images of CdSe Tps. 

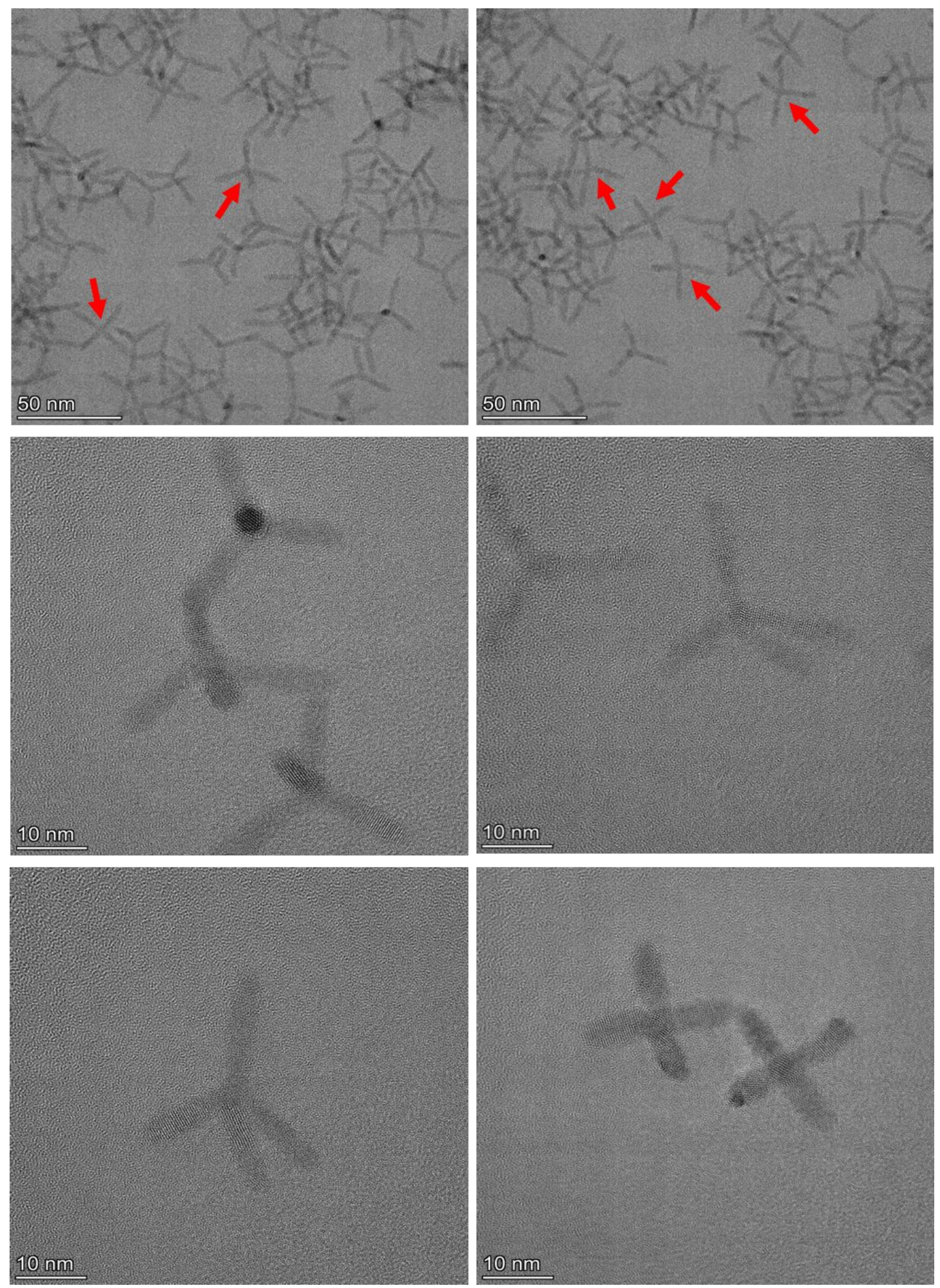

Fig. S3 Additional TEM images of Tps. 


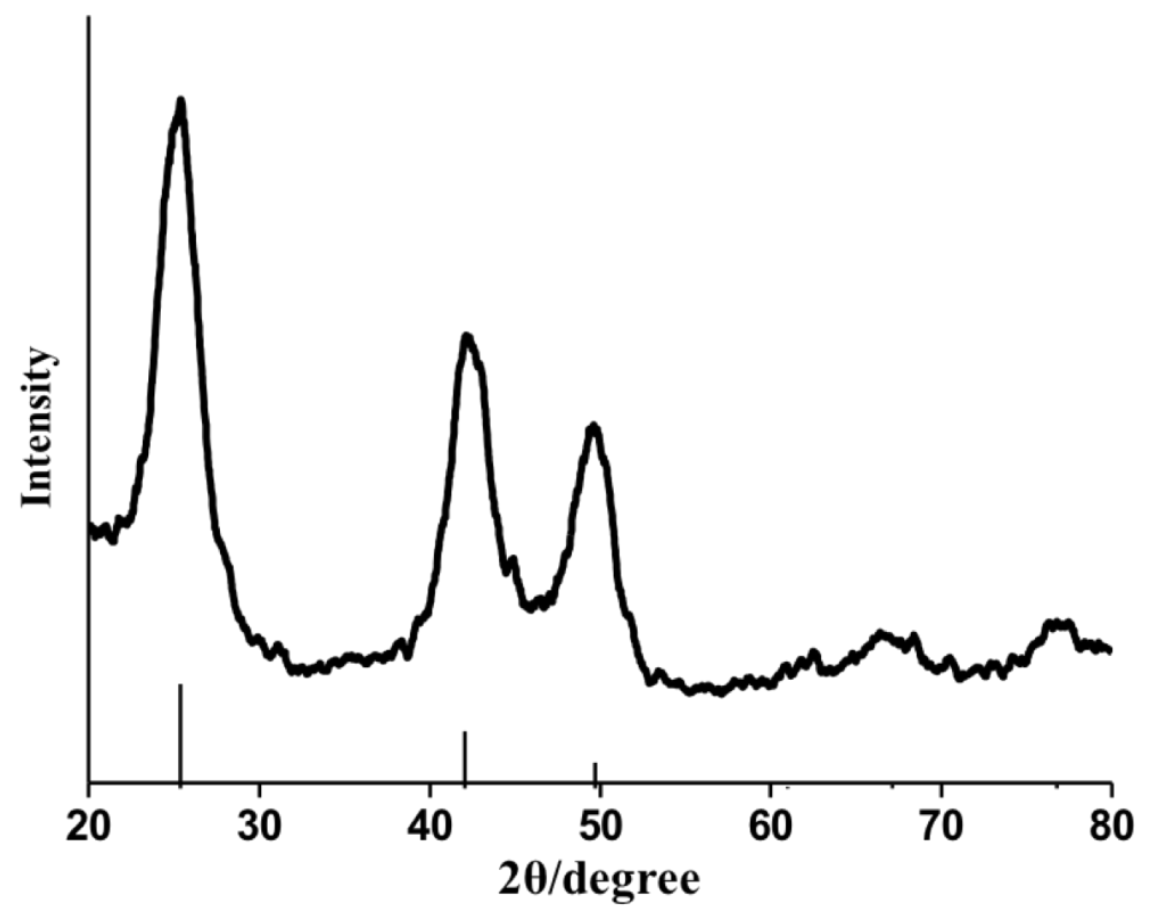

Fig. S4 XRD of ZB CdSe seed (Tp0).

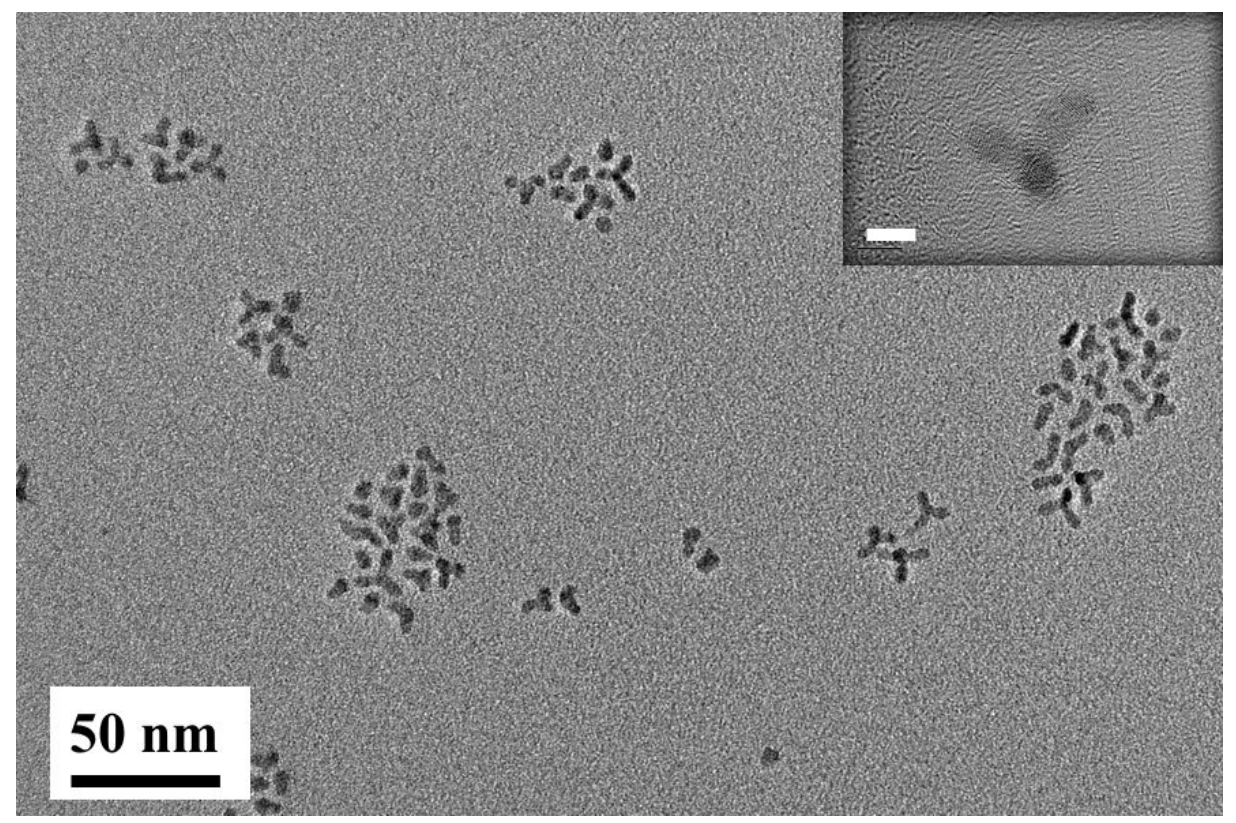

Fig. S5 TEM image of ZB CdSe seed and Tps. Inset: the magnified TEM image of an individual tetrapod. The scale bar in the inset is $5 \mathrm{~nm}$. 


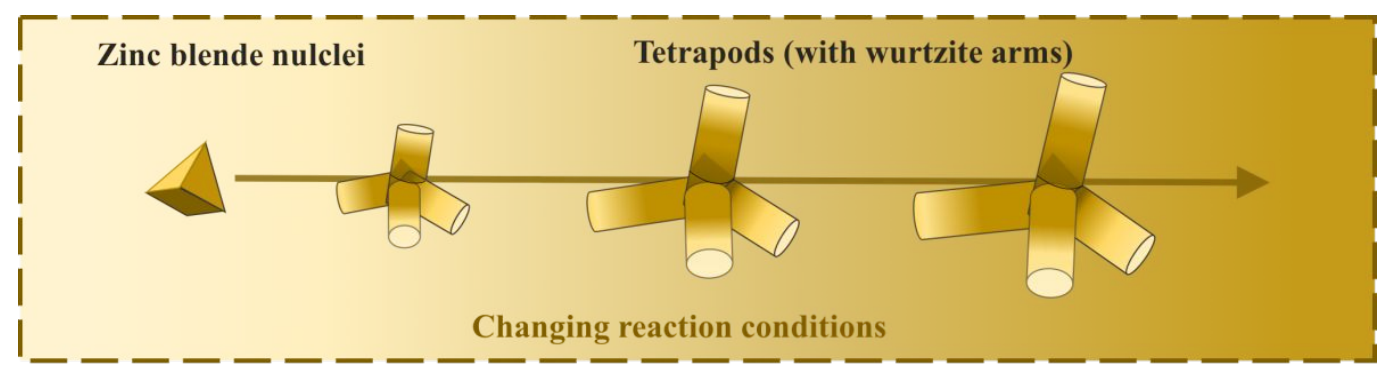

Scheme S1 Schematic illustration of the growth mechanism of CdSe tetrapods. The left particle represents the CdSe ZB core; the right NCs represent the CdSe Tps with different arm size.
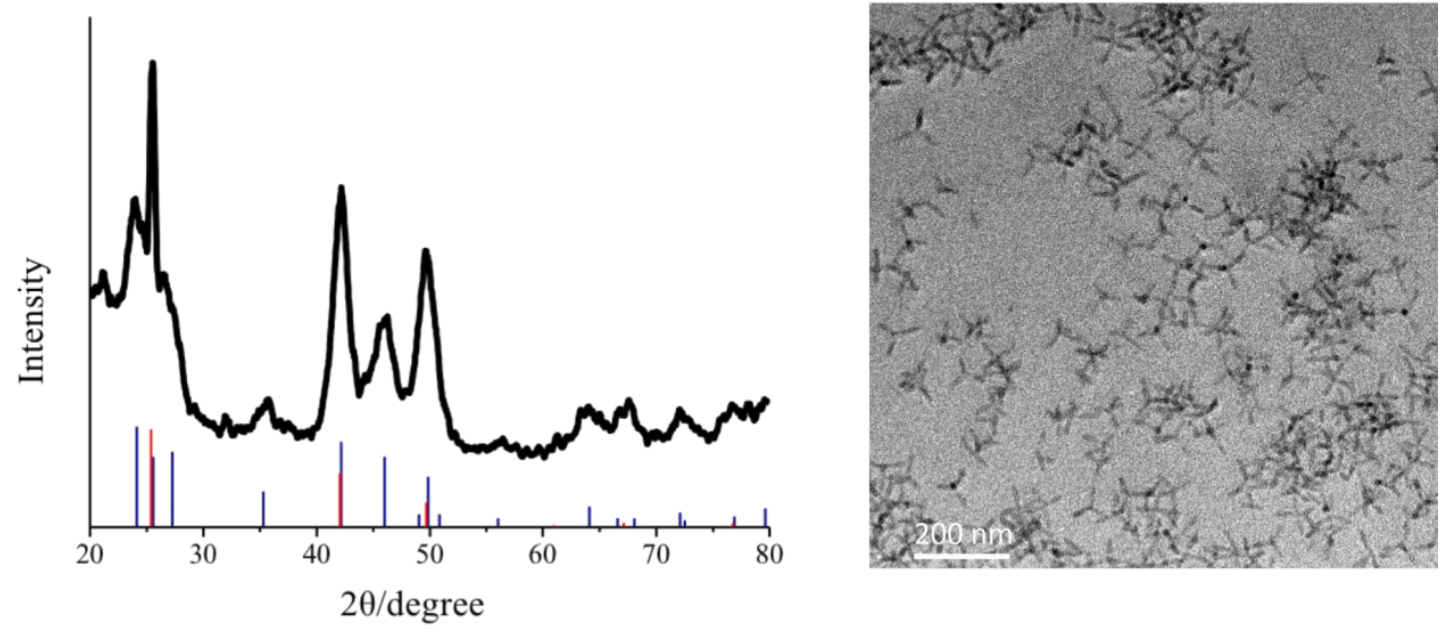

Fig. S6 XRD and TEM image of L-Cys capped CdSe Tp3. 


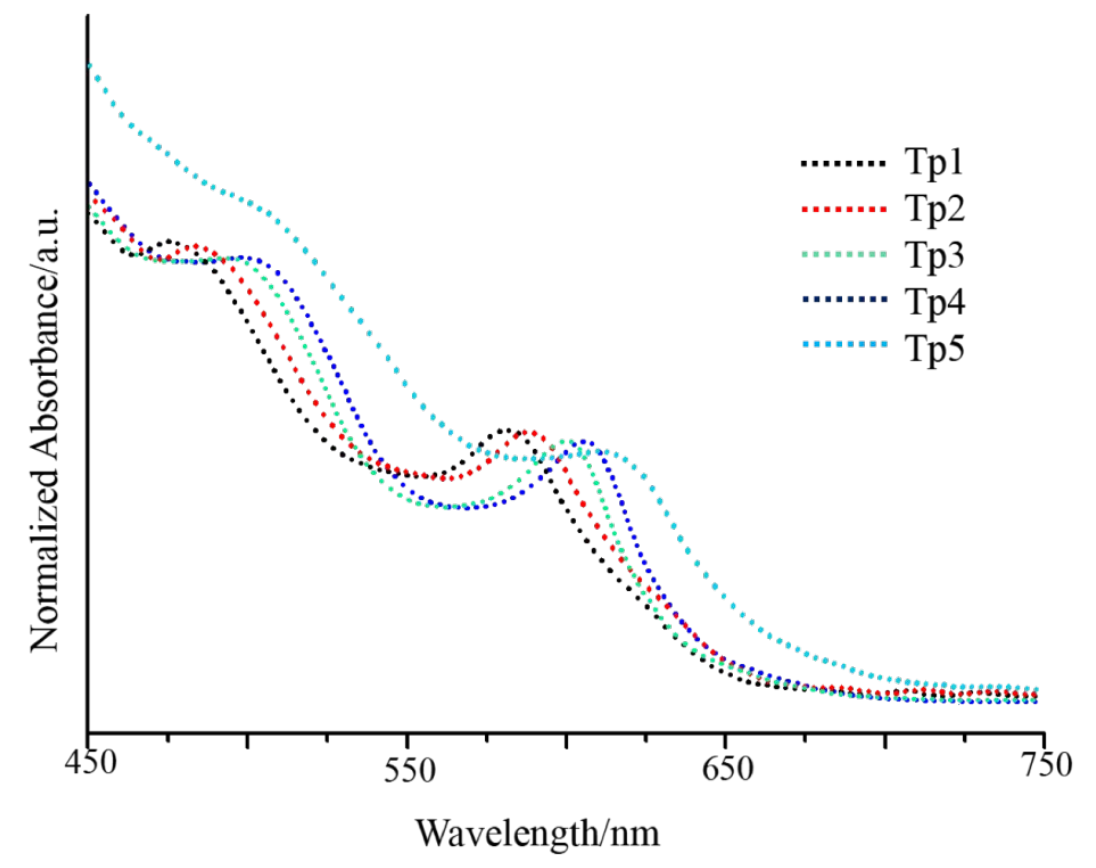

Fig. S7 The normalized absorption spectra (normalized at the band gap absorption) for Tp1 Tp5 after ligand exchange with L-Cys.

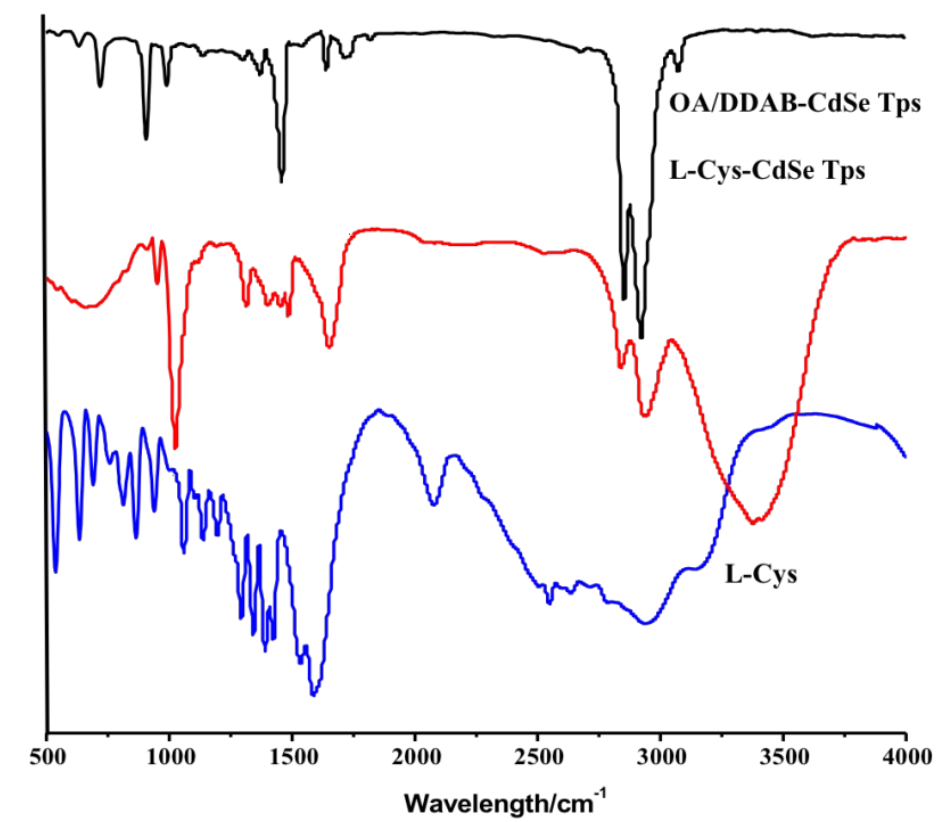

Fig. S8 FTIR spectra of CdSe Tp5 stabilized with OA/DDAB (black) and L-cysteine (red). For comparison, the FTIR spectra of free L-cysteine was measured (Blue). 
a
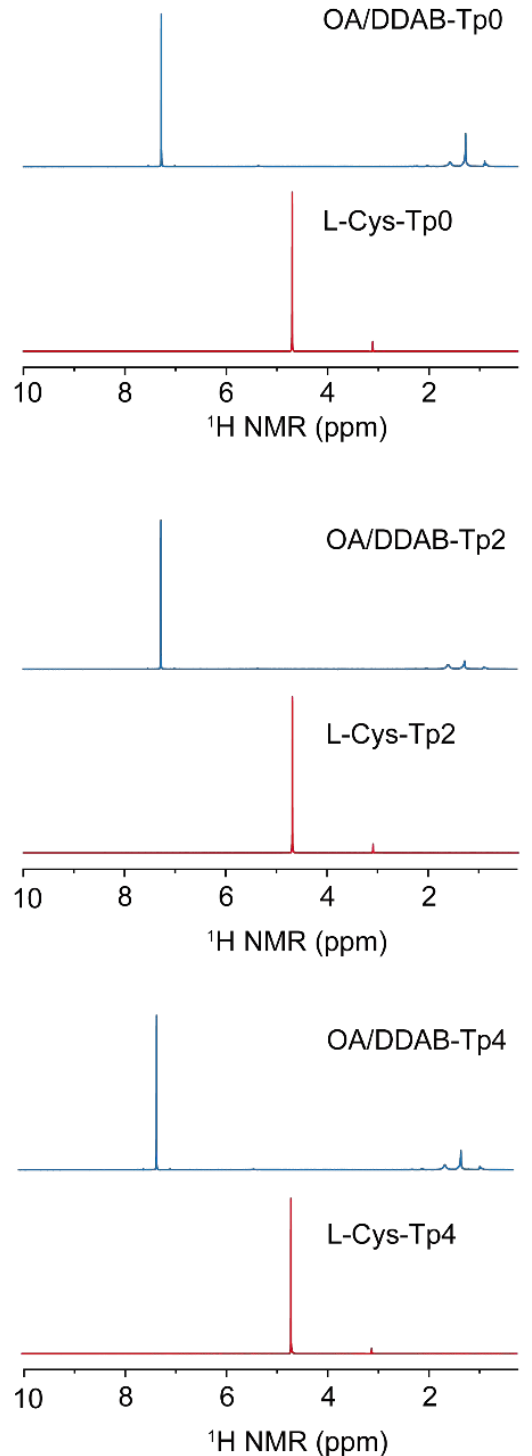

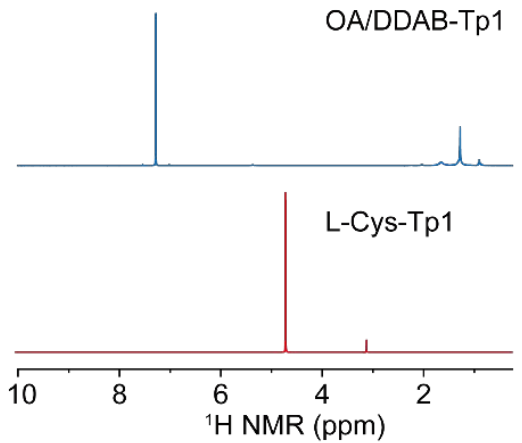

d

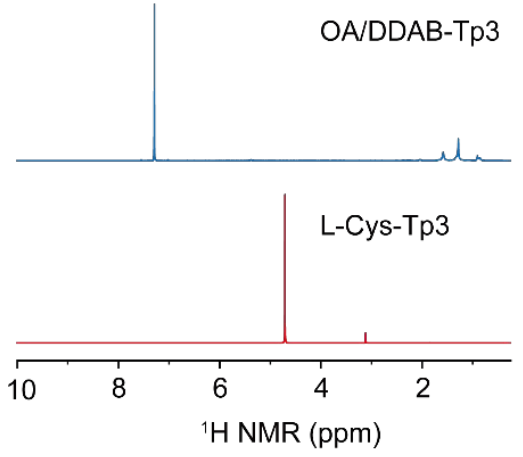

f

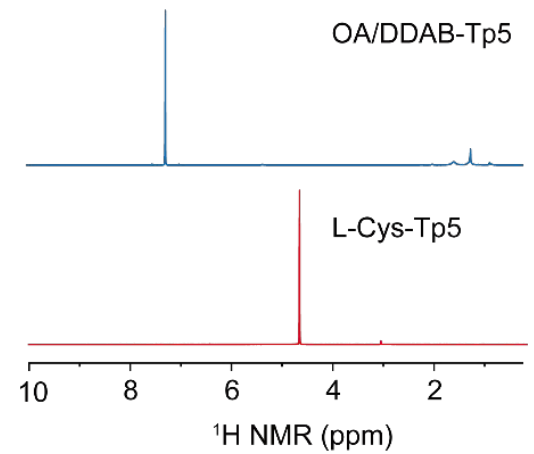

Fig. S9 ${ }^{1} \mathrm{H}$ NMR spectra of Tp0-5 before (blue) and after (red) ligand exchange by Lcysteine. Note: $7.3 \mathrm{ppm}$ and $4.7 \mathrm{ppm}$ are from the solvent chloroform- $\mathrm{d}$ and $\mathrm{D}_{2} \mathrm{O}$. 


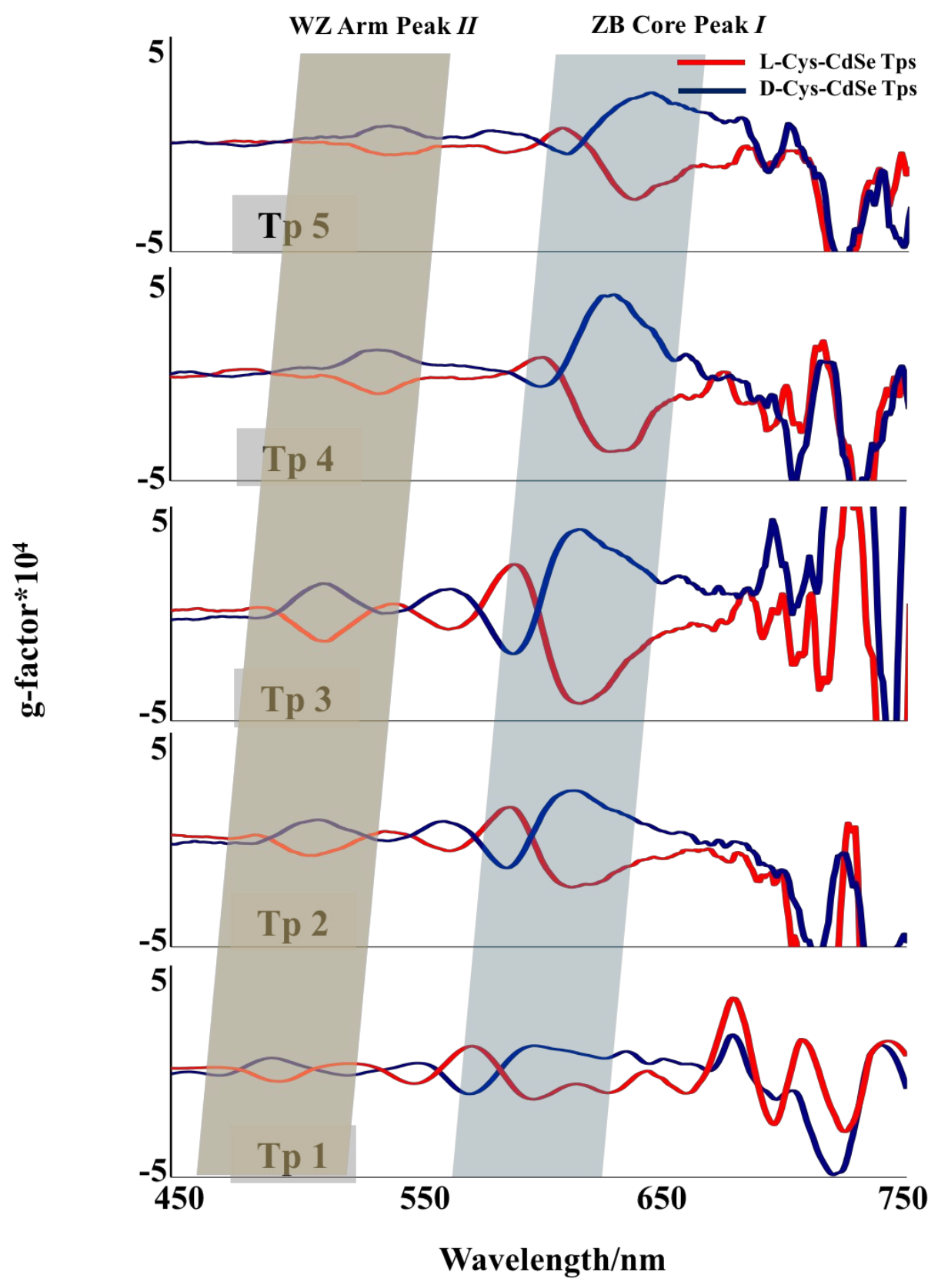

Fig. S10 G-factor graphs of L-Cys (red) and D-Cys (blue) functionalised CdSe Tps with varying AL (marked Tp1 Tp5). 
Table S2 Peak I CD anisotropic factors of L-Cys-CdSe Tps

\begin{tabular}{cccc}
\hline L-Cys-TPs & $\mathbf{g}_{\mathbf{C D}}{ }^{+} / \lambda_{\mathbf{C D}}(\mathbf{n m})^{\mathbf{a}}$ & $\mathbf{g}_{\mathbf{C D}}{ }^{-} / \lambda_{\mathbf{C D}}(\mathbf{n m})^{\mathbf{b}}$ & $\left|\mathbf{g}_{\mathbf{C D}}{ }^{+}-\mathbf{g}_{\mathbf{C D}}{ }^{-}\right| / \mathbf{2}^{\mathbf{c}}$ \\
\hline Tp 1 & $1.19 \times 10^{-4} / 573$ & $-1.32 \times 10^{-4} / 599$ & $1.26 \times 10^{-4}$ \\
Tp 2 & $1.51 \times 10^{-4} / 587$ & $-2.18 \times 10^{-4} / 612$ & $1.85 \times 10^{-4}$ \\
Tp 3 & $2.29 \times 10^{-4} / 589$ & $-4.18 \times 10^{-4} / 616$ & $3.24 \times 10^{-4}$ \\
Tp 4 & $7.50 \times 10^{-5} / 610$ & $-3.63 \times 10^{-4} / 638$ & $2.19 \times 10^{-4}$ \\
Tp 5 & $6.74 \times 10^{-5} / 609$ & $-2.83 \times 10^{-4} / 640$ & $1.49 \times 10^{-4}$ \\
\hline
\end{tabular}

Table S3 Peak I CD anisotropic factors of D-Cys-CdSe Tps

\begin{tabular}{cccc}
\hline D-Cys-TPs & $\mathbf{g}_{\mathbf{C D}}{ }^{+} / \lambda_{\mathbf{C D}}(\mathbf{n m})^{\mathbf{a}}$ & $\mathbf{g}_{\mathbf{C D}}{ }^{-} / \lambda_{\mathbf{C D}}(\mathbf{n m})^{\mathbf{b}}$ & $\left|\mathbf{g}_{\mathbf{C D}}{ }^{+}-\mathbf{g}_{\mathbf{C D}}{ }^{-}\right| / \mathbf{2}^{\mathbf{c}}$ \\
\hline Tp 1 & $1.20 \times 10^{-4} / 599$ & $-1.07 \times 10^{-4} / 571$ & $1.14 \times 10^{-4}$ \\
Tp 2 & $2.29 \times 10^{-4} / 614$ & $-1.31 \times 10^{-4} / 587$ & $1.80 \times 10^{-4}$ \\
Tp 3 & $3.94 \times 10^{-4} / 616$ & $-1.87 \times 10^{-4} / 589$ & $2.91 \times 10^{-4}$ \\
Tp 4 & $3.70 \times 10^{-4} / 640$ & $-5.68 \times 10^{-5} / 609$ & $2.13 \times 10^{-4}$ \\
Tp 5 & $2.75 \times 10^{-4} / 645$ & $-4.15 \times 10^{-5} / 611$ & $1.28 \times 10^{-4}$ \\
\hline
\end{tabular}

${ }^{\mathrm{a}, \mathrm{b}} \mathrm{CD}$ anisotropy $\mathrm{g}_{\mathrm{CD}}$ factors at the most intense positive and negative $\mathrm{CD}$ bands (nm).

${ }^{c}$ Magnitude of the $g_{C D}$ factor, defined as $\left|g_{C^{+}}{ }^{+}-g_{C D}{ }^{-}\right| / 2$.

Table S4 Peak II CD anisotropic factors of L/D-Cys-CdSe Tps (second peak)

\begin{tabular}{c|ccc}
\hline Cys-TPs & $\begin{array}{c}\text { Wavelength } \\
\text { /nm }\end{array}$ & D-Cys-g $_{\mathbf{C D}}{ }^{+}$ & L-Cys-g $^{-}$ \\
\hline Tp 1 & 491 & $6.29 \times 10^{-5}$ & $-5.50 \times 10^{-5}$ \\
Tp 2 & 509 & $9.25 \times 10^{-5}$ & $-8.28 \times 10^{-5}$ \\
Tp 3 & 511 & $1.40 \times 10^{-4}$ & $-1.36 \times 10^{-4}$ \\
Tp 4 & 535 & $1.10 \times 10^{-4}$ & $-9.89 \times 10^{-5}$ \\
Tp 5 & 540 & $7.56 \times 10^{-5}$ & $-5.45 \times 10^{-5}$ \\
\hline
\end{tabular}



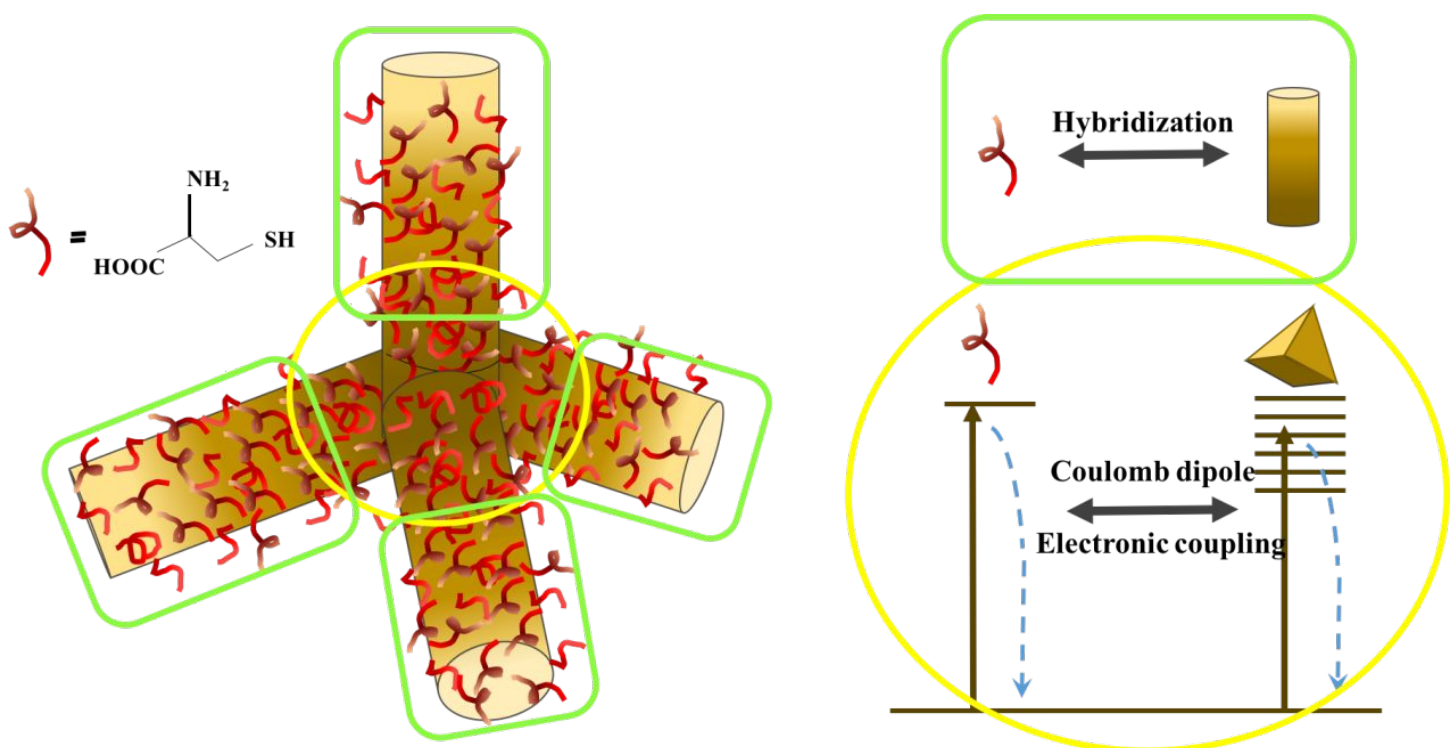

Scheme S2 Illustration of the chirality mechanisms for the L-Cys-CdSe Tps. 